\title{
Autoavaliação da saúde por idosos brasileiros: revisão sistemática da literatura
}

\author{
Valéria Pagotto, ${ }^{1}$ Maria Márcia Bachion ${ }^{1}$ e \\ Erika Aparecida da Silveira ${ }^{2}$
}

Como citar Pagotto V, Bachion MM, Silveira EA. Autoavaliação da saúde por idosos brasileiros: revisão sistemática da literatura. Rev Panam Salud Publica. 2013;33(4):302-10.

RESUMO Objetivo. Realizar uma revisão sistemática da literatura sobre autoavaliação do estado de saúde na população idosa brasileira.

Métodos. Foram pesquisadas as bases de dados Medline e LILACS, conforme a metodologia PRISMA (Preferred Reporting Items for Systematic Reviews and Meta-Analyses). Foram incluídos artigos originais sobre a autoavaliação do estado de saúde realizados com idosos brasileiros. Foram consideradas as características gerais dos estudos, a prevalência de autoavaliação negativa da saúde, os fatores associados a essa autoavaliação negativa, a pergunta utilizada para a consulta e as categorias de resposta.

Resultados. Dos 97 estudos encontrados, 11 atenderam os critérios de inclusão. Foram identificadas variações entre os estudos quanto à formulação da pergunta e às opções de resposta. A prevalência de autoavaliação negativa de saúde variou de 12,6 a 51,9\% entre os estudos. As variáveis dependentes predominantemente associadas a autoavaliação negativa de saúde foram: presença de doenças, número de medicamentos em uso, renda familiar/domiciliar mensal, internações, consultas médicas, dificuldade/incapacidade para atividades de vida diária, presença de sintomas depressivos e ansiosos e queixa de insônia.

Conclusões. A heterogeneidade de fatores associados à autoavaliação negativa indica que a saúde dos idosos é definida por determinantes que se aproximam do conceito ampliado de saúde. É recomendável a padronização das perguntas e respostas de pesquisa sobre autoavaliação de saúde em idosos, já que essas informações possibilitarão conhecer, acompanhar e comparar resultados para orientar a tomada de decisão no tocante à formulação de políticas de saúde para a Brasil e a América Latina.

Palavras-chave Idoso; autoavaliação; nível de saúde; revisão; Brasil.

Nos inquéritos populacionais, a avaliação da saúde pode englobar três domínios de investigação: saúde "testada", avaliada por meio de exames laborato-

\footnotetext{
Universidade Federal de Goiás, Faculdade de Enfermagem, Goiânia (GO), Brasil. Correspondência: Valéria Pagotto, valeriapagotto@gmail.com

2 Universidade Federal de Goiás, Pró-Reitoria de Pesquisa e Pós-Graduação, Goiânia (GO), Brasil.
}

riais e funcionais; saúde "observada", baseada na avaliação clínica feita por profissionais especializados; e saúde "percebida", pautada na autoavaliação da saúde, nos conhecimentos e nas crenças pessoais de cada indivíduo $(1,2)$. Nos últimos anos, a autoavaliação tem sido utilizada em inquéritos populacionais em todo o mundo (3-5), pois está fortemente associada ao estado "real" ou "objetivo" de saúde das pessoas, incorporando seus aspectos físicos, cognitivos e emocionais. Por ser uma medida de autorreferência, é facilmente analisada em amostra ampla da população, de forma rápida, acessível e econômica, pois é obtida por meio de uma única pergunta $(6,7)$. Além disso, a autoavaliação do estado de saúde é 
um indicador recomendado pela Organização Mundial da Saúde (OMS) para verificar a saúde das populações.

$\mathrm{O}$ franco processo de envelhecimento da população, inclusive no Brasil, torna imperativas as investigação acerca da saúde dos idosos - já que, historicamente, nunca houve nas sociedades um grupo tão grande de pessoas com idade tão avançada (8). Nesse contexto, a influência de variáveis socioeconômicas, demográficas, condições de saúde e estilo de vida, entre outras, tem sido investigada em alguns estudos sobre autoavaliação de saúde com idosos brasileiros (9-11). Entretanto, esses estudos têm produzido resultados controversos, talvez em virtude das diferenças na forma de elaborar a questão e nas opções de resposta, sendo necessária uma uniformização para melhorar a comparação entre os resultados das pesquisas $(6,9)$.

Há evidências do poder preditivo da autoavaliação do estado de saúde quanto à morbimortalidade e ao declínio funcional em idosos de ambos os sexos (12). Diante disso, conhecer os fatores que influenciam na percepção de saúde dos idosos poderá contribuir para identificar quais dimensões da saúde precisam ser fortalecidas para que esse grupo tenha atenção adequada às suas necessidades.

Sendo assim, o objetivo deste estudo foi realizar uma revisão sistemática da literatura sobre autoavaliação do estado de saúde na população idosa brasileira.

\section{MATERIAS E MÉTODOS}

Trata-se de um estudo de revisão sistemática, conduzido conforme a metodologia Preferred Reporting Items for Systematic Reviews and Meta-Analyses (PRISMA) (13). Para identificar os artigos acerca do assunto, realizou-se busca nas bases Medline e LILACS, de setembro a outubro de 2011, com a seguinte estratégia de busca: aged or elderly or aging or aging and health and self and rated and health or self and evaluation and of and health and status or health and status and health and inequalities or health and services and coverage or determinants and of and health and inequalities. Somente foram utilizados termos em inglês. Buscas manuais foram feitas nas referências bibliográficas dos artigos encontrados.

Para a inclusão dos artigos, foram empregados os seguintes critérios: estudos observacionais, cujo desfecho era a auto- avaliação de saúde investigada por meio de pergunta autoaplicada ou aplicada pelo entrevistador, nos quais os objetivos incluíssem a prevalência do desfecho e/ ou os fatores associados, com metodologia claramente descrita, com população alvo de idosos, realizados em cenários do território brasileiro, publicados em periódicos na língua inglesa, portuguesa ou espanhola, no período de 1999 a 2011, com textos disponíveis na íntegra. Foram excluídos estudos cujo desfecho era autoavaliação de saúde bucal ou mental.

Após a consulta às bases de dados e a aplicação das estratégias de busca, foram identificados estudos que apresentavam duplicidade entre as bases. Foram lidos todos os resumos resultantes. Nos casos em que a leitura do resumo não era suficiente para estabelecer se o artigo deveria ser incluído, considerando-se os critérios de inclusão definidos, o artigo foi lido na íntegra para determinar sua elibigilidade. Quando o resumo era suficiente, os artigos eram selecionados e então obtida a versão integral para confirmação de elegibilidade e inclusão no estudo.

Para extração dos dados dos artigos, elaborou-se um instrumento contendo as seguintes informações: autores, ano de publicação, local de publicação, tipo de estudo, tamanho da amostra, forma de avaliação do desfecho (pergunta, opções de resposta e categorização), prevalência do desfecho, fatores associados à autoavaliação de saúde e tipo de análise estatística (13).

A análise dos estudos encontrados foi feita de forma descritiva e realizada em duas etapas. A primeira incluiu: ano, autoria, local do estudo, tipo de estudo, população-alvo, delineamento do estudo, forma de avaliação do desfecho quanto à elaboração da pergunta e opções de resposta. A segunda etapa compreendeu a prevalência do desfecho analisado e os fatores associados a esse desfecho (13).

\section{RESULTADOS}

Após eliminação de 12 artigos duplicados, foram selecionados 86 artigos. Desses, 52 foram excluídos após a análise dos títulos e resumos. Dos 34 artigos elegíveis, 23 foram excluídos pelos seguintes motivos: em 15 a autoavaliação do estado de saúde era variável de exposição; seis não se referiam à população idosa; um estudo era de revisão sistemática; e um artigo era descritivo. Ao final, 11 estudos foram incluídos na presente revisão sistemática (9-11, 14-21), dos quais três continham pessoas com 18 anos ou mais, incluindo a população idosa $(18,19,21)$. Esses foram incluídos por apresentar prevalência estratificada por faixa etária e abranger grande amostra de pessoas acima de 60 na população brasileira (PNAD e VIGITEL). Não foram encontrados estudos por meio da busca manual nas referências dos artigos encontrados. A figura 1 apresenta a síntese do processo de seleção dos artigos.

Quanto às características gerais, a publicação mais antiga era de 2004; oito artigos tratavam da Região Sudeste, um da Região Centro-Oeste e dois tinham abrangência nacional. $\mathrm{O}$ delineamento do tipo transversal foi predominante $(63,3 \%)$, e as amostras variaram de 230 a 2876 idosos (tabela 1).

Quanto à forma de avaliação do desfecho, observaram-se diversificações quanto à elaboração da pergunta e ao número de opções de resposta, que variou entre quatro e cinco opções (tabela 2). Além disso, as categorias de resposta empregadas, apesar de semelhantes, não eram exatamente iguais: seis estudos utilizaram a categoria "muito boa" e quatro usaram o termo "excelente"; para o segundo nível da autoavaliação de saúde, a categoria foi "boa" em oito pesquisas e "muito boa" em dois estudos. Isso implicou diversidade também no terceiro nível, que aparece como "razoável" em seis pesquisas. A pior avaliação foi "ruim" no caso dos estudos que utilizaram quatro opções de resposta, ou "muito ruim" e "péssimo" quando havia cinco opções de resposta.

O desfecho foi construído agrupandose as categorias e obtendo-se uma variável dicotômica. A categoria "razoável" ora foi agrupada entre as opções de respostas positivas (boa, muito boa), ora entre as negativas (ruim, muito ruim). Todos os estudos enfocaram a autoavaliação negativa do estado de saúde.

A prevalência da autoavaliação do estado de saúde como ruim variou de 12,6 a 51,9\% (figura 2). Em três estudos a prevalência foi superior a $30 \%$.

Quanto às variáveis dependentes utilizadas (tabela 3), todas se referiam a condições socioeconômicas, demográficas e de saúde. O estudo da linha de base do Projeto Bambuí foi o que usou o maior 
FIGURA 1. Fluxograma de identificação e seleção dos artigos para revisão sistemática sobre autoavaliação do estado de saúde de idosos, Brasil, 1999 a 2011

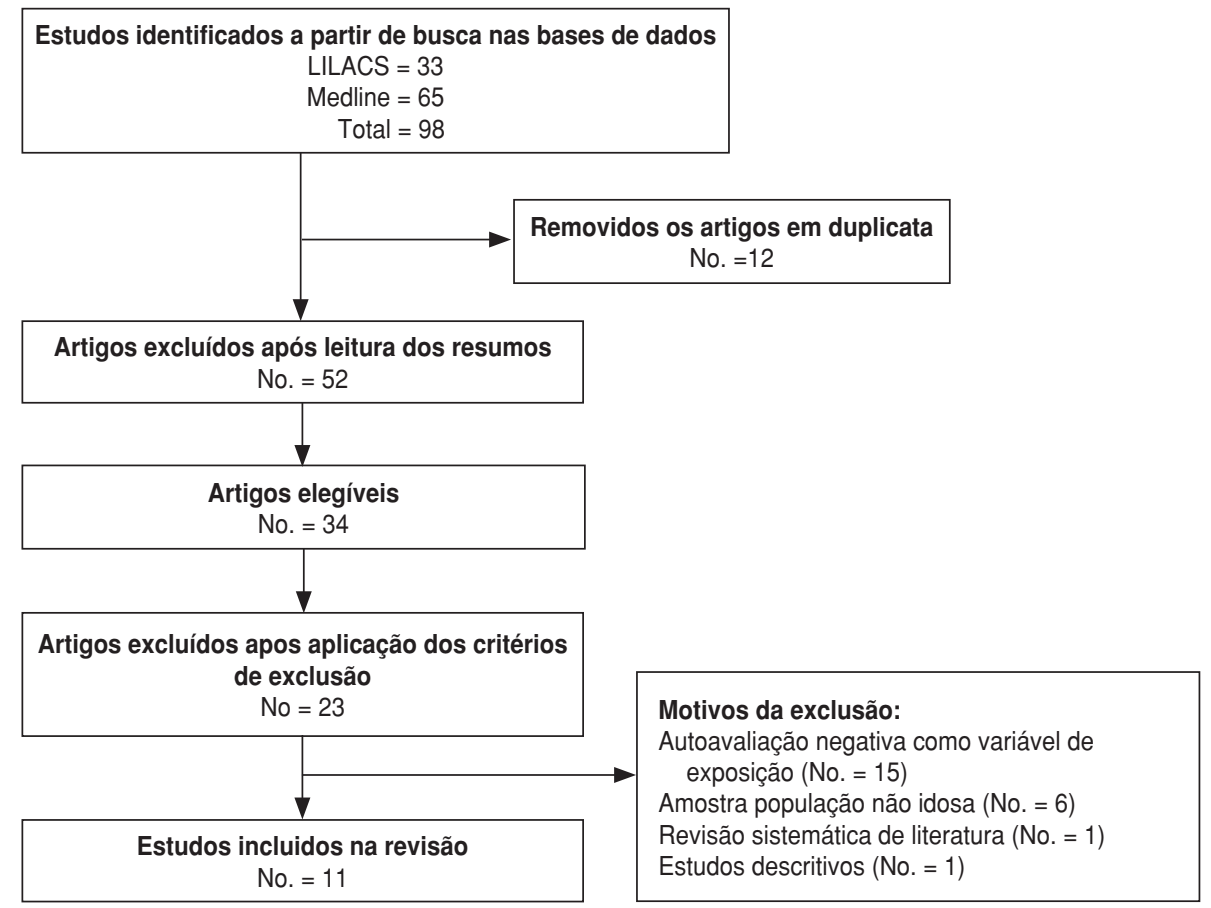

TABELA 1. Características dos estudos sobre auto-avaliação do estado de saúde em idosos segundo autor, ano, local, desenho do estudo e amostra, Brasil, 1999 a 2011

\begin{tabular}{|c|c|c|c|c|}
\hline Código & Autor, ano (referência) & Local & Desenho & Amostra (no.) \\
\hline 1 & Lima-Costa et al., 2004 (9) & Bambuí, Estado de Minas Gerais & Coorte & 1516 \\
\hline 2 & Lima-Costa et al., 2005 (14) & Bambuí, Estado de Minas Gerais & Coorte & 1516 \\
\hline 3 & Szwarcwald et al., $2005(21)^{\mathrm{a}}$ & Brasil (inquérito mundial de saúde) & Transversal & 4997 pessoas $\geq 18$ anos \\
\hline \multirow[t]{2}{*}{5} & Lima-Costa et al., 2007 (15) & Bambuí, Estado de Minas Gerais (PNAD) & Coorte (Bambuí) & Bambuí: 1516 \\
\hline & & & Transversal (PNAD) & PNAD 1998: 28943 \\
\hline 6 & Souza et al., $2008(18)^{a}$ & $\begin{array}{l}18 \text { capitais brasileiras } \\
(\text { IDANT) })^{\mathrm{b}}\end{array}$ & Transversal & 26424 pessoas $\geq 15$ anos $^{d}$ \\
\hline 8 & Barros et al., $2009(19)^{\mathrm{a}}$ & Capitais brasileiras e Distrito Federal (VIGITEL) ${ }^{\mathrm{C}}$ & Transversal & 54369 pessoas $\geq 18$ anos $^{d}$ \\
\hline 9 & Santiago et al., 2010 (16) & Juiz de Fora, Estado de Minas Gerais & Transversal & 2876 homens \\
\hline 10 & Jardim et al., 2010 (17) & $\begin{array}{l}\text { Belo Horizonte, Estado de Minas Gerais } \\
\text { (Projeto Saúde e Envelhecimento) }\end{array}$ & Transversal & 230 \\
\hline 11 & Pagotto et al., 2011 (20) & Goiânia, Estado de Goiás (Projeto Idoso/Goiânia) & Transversal & 403 \\
\hline
\end{tabular}

a Foram utilizados os dados de prevalência dos indivíduos com 60 anos ou mais. Não foram avaliados os fatores associados, pois abrangiam a população total.

b Inquérito Domiciliar sobre Comportamentos de Risco e Morbidade Referida de Doenças e Agravos não transmissíveis.

c VIGITEL = Vigilância de Fatores de Risco e Proteção para Doenças Crônicas por Inquérito Telefônico.

d Os autores não descrevem na metodologia qual o número total de idosos da amostra. Foram utilizados apenas os dados de prevalência, pois os fatores associados foram analisados para o universo total da amostra, que contempla pessoas jovens, adultas e idosas.

número de variáveis. Nos estudos incluídos, as variáveis predominantemente associadas ao desfecho "autoavaliação da saúde como ruim" foram: presença de doenças (quatro estudos), número de medicamentos em uso, renda familiar/ domiciliar mensal (três estudos), internações, consultas médicas, dificuldade/ incapacidade para atividades de vida diária e presença de sintomas depressivos e ansiosos, bem como queixa de insônia (dois estudos).

\section{DISCUSSÃO}

Conforme a presente revisão sistemática da literatura, a autoavaliação de saúde foi empregada no Brasil como variável desfecho pela primeira vez na 
TABELA 2. Características da pergunta sobre auto-avaliação do estado de saúde em idosos, opções de resposta, categorização da variável e construção do desfecho, Brasil, 1999 a 2011

\begin{tabular}{|c|c|c|c|c|c|}
\hline Código & $\begin{array}{l}\text { Estudo, ano } \\
\text { (referência) }\end{array}$ & Pergunta & $\begin{array}{l}\text { Opções de } \\
\text { resposta }\end{array}$ & Categorização da variável & Desfecho \\
\hline 1 & $\begin{array}{l}\text { Lima-Costa } \\
\text { et al., } 2004 \text { (9) }\end{array}$ & Você diria que sua saúde é: & $\begin{array}{l}\text { - } \text { Muito boa } \\
\text { - } \text { Boa } \\
\text { - Razoável } \\
\text { - Ruim } \\
\text { - Muito ruim }\end{array}$ & $\begin{array}{l}\text { - Razoável/boa/muito boa } \\
\text { - Ruim/muito ruim }\end{array}$ & Ruim/muito ruim \\
\hline 2 & $\begin{array}{l}\text { Lima-Costa } \\
\text { et al., } 2005 \text { (14) }\end{array}$ & Você diria que sua saúde é: & $\begin{array}{l}\text { - Muito boa } \\
\text { - Boa } \\
\text { - Razoável } \\
\text { - Ruim } \\
\text { - Muito ruim }\end{array}$ & $\begin{array}{l}\text { - Razoável/boa/muito boa } \\
\text { - Ruim/muito ruim }\end{array}$ & $\begin{array}{l}\text { Auto-percepção de saúde ruim/muito } \\
\text { ruim de idosos com baixa renda }\end{array}$ \\
\hline 3 & $\begin{array}{l}\text { Szwarcwald } \\
\text { et al., } 2005(21)\end{array}$ & $\begin{array}{l}\text { Em geral, como o(a) senhor(a) avalia } \\
\text { a sua saúde atualmente? }\end{array}$ & $\begin{array}{l}\text { - } \text { Muito boa } \\
\text { - } \text { Boa } \\
\text { - } \text { Moderada } \\
\text { - } \text { Ruim } \\
\text { - } \text { Muito ruim }\end{array}$ & $\begin{array}{l}\text { - Muito boa/boa } \\
\text { - Moderada } \\
\text { - Ruim/muito ruim }\end{array}$ & Ruim/muito ruim \\
\hline 4 & $\begin{array}{l}\text { Alves e } \\
\text { Rodrigues, } 2005 \\
(10)\end{array}$ & Você diria que sua saúde é: & $\begin{array}{l}\text { - Excelente } \\
\text { - Muito boa } \\
\text { - Boa } \\
\text { - Regular } \\
\text { - Ruim }\end{array}$ & $\begin{array}{l}\text { Auto-percepção de saúde boa: } \\
\text { excelente/muito boa/ boa } \\
\text { Auto-percepção de saúde } \\
\text { ruim: regular e ruim }\end{array}$ & Auto-percepção de saúde ruim \\
\hline 5 & $\begin{array}{l}\text { Lima-Costa } \\
\text { et al., } 2007 \text { (15) }\end{array}$ & $\begin{array}{l}\text { De uma maneira geral você diria que } \\
\text { a sua saúde é: }\end{array}$ & $\begin{array}{l}\text { - } \text { Muito boa } \\
\text { - } \text { Boa } \\
\text { - Razoável } \\
\text { - Ruim } \\
\text { - Muito ruim }\end{array}$ & $\begin{array}{l}\text { - Razoável/boa/muito boa } \\
\text { - Ruim/muito ruim }\end{array}$ & Ruim/muito ruim \\
\hline 6 & $\begin{array}{l}\text { Souza et al., } \\
2008(18)\end{array}$ & $\begin{array}{l}\text { De um modo geral, em comparação } \\
\text { com pessoas da sua idade, como } \\
\text { o(a) Sr.(a) considera o seu próprio } \\
\text { estado de saúde? }\end{array}$ & $\begin{array}{l}\text { - Excelente } \\
\text { - Muito bom } \\
\text { - Bom } \\
\text { - Regular } \\
\text { - Ruim }\end{array}$ & $\begin{array}{l}\text { - Excelente/muito bom/bom } \\
\text { - Regular/ Ruim }\end{array}$ & $\begin{array}{l}\text { Auto-avaliação de saúde regular } \\
\text { ou ruim }\end{array}$ \\
\hline 7 & $\begin{array}{l}\text { Souza e Silver, } \\
2008(11)\end{array}$ & Não descrita no corpo do texto & $\begin{array}{l}\text { - Não descrito } \\
\text { no corpo do } \\
\text { texto }\end{array}$ & Não descrito no corpo do texto & Ruim/ péssima \\
\hline 8 & $\begin{array}{l}\text { Barros et al., } \\
2009(19)\end{array}$ & $\begin{array}{l}\text { O senhor classificaria seu estado de } \\
\text { saúde como: }\end{array}$ & $\begin{array}{l}\text { - Excelente } \\
\text { - Bom } \\
\text { - Regular } \\
\text { - Ruim }\end{array}$ & $\begin{array}{l}\text { - Excelente/bom/regular } \\
\text { - Ruim }\end{array}$ & Auto-avaliação de saúde ruim \\
\hline 9 & $\begin{array}{l}\text { Santiago et al., } \\
2010(16)\end{array}$ & Como é a sua saúde de forma geral? & $\begin{array}{l}\text { - Excelente } \\
\text { - Boa } \\
\text { - Razoável } \\
\text { - Ruim }\end{array}$ & $\begin{array}{l}\text { - Excelente/boa } \\
\text { - Razoável/ruim }\end{array}$ & $\begin{array}{l}\text { Auto-avaliação de saúde razoável/ } \\
\text { ruim }\end{array}$ \\
\hline 10 & $\begin{array}{l}\text { Jardim et al., } \\
2010(17)\end{array}$ & $\begin{array}{l}\text { Idoso: De uma maneira geral, você } \\
\text { considera sua saúde } \\
\text { Informante: De uma maneira geral, } \\
\text { você considera a saúde do(a) Sr(a). } \\
\text { [nome do idoso]: }\end{array}$ & $\begin{array}{l}\text { - Muito boa } \\
\text { - Boa } \\
\text { - Razoável } \\
\text { - Ruim } \\
\text { - Muito ruim }\end{array}$ & $\begin{array}{l}\text { - Razoável/boa/muito boa } \\
\text { - Ruim/muito ruim }\end{array}$ & $\begin{array}{l}\text { Ruim/muito ruim tanto para idoso } \\
\text { quanto para informante }\end{array}$ \\
\hline 11 & $\begin{array}{l}\text { Pagotto et al., } \\
2011(20)\end{array}$ & $\begin{array}{l}\text { O que o } \mathrm{Sr}(\mathrm{a}) \text {. acha do seu estado de } \\
\text { saúde no último mês? }\end{array}$ & $\begin{array}{l}\text { - Muito Bom } \\
\text { - Bom } \\
\text { - Regular } \\
\text { - Fraco } \\
\text { - Péssimo }\end{array}$ & $\begin{array}{l}\text { - Muito bom/bom/regular } \\
\text { - Fraco/péssimo }\end{array}$ & Autoavaliação de saúde ruim \\
\hline
\end{tabular}

PNAD, em 1998, para avaliar a percepção negativa de saúde da população em geral (6). Já para a população idosa, esse desfecho foi estudado pela primeira vez em 2004, no Projeto Bambuí (9). Observamos ainda uma predominância dos estudos que utilizaram a autoavaliação negativa de saúde como variável de exposição, o que demonstra o interesse em utilizar essa variá- vel como possível preditora de outros desfechos.

Até o momento, são poucas as publicações nacionais sobre a autoavaliação do estado de saúde de idosos, estando a maior parte concentrada no Sudeste (9-11, 14-17), impossibilitando comparações entre as regiões. Embora não haja dados de todas as regiões brasileiras, há um estudo com pessoas acima de 50 anos
(18) que investigou a saúde autoavaliada como ruim em algumas capitais brasileiras. Nesse estudo, pode-se verificar que a prevalência de autoavaliação negativa difere entre as regiões, com valores mais elevados no Nordeste, com homogeneidade interna nas regiões Norte/ Nordeste e Sul/Sudeste e uma heterogeneidade entre essas regiões. Dados do VIGITEL (19), que abrange as capitais 
FIGURA 2. Prevalência da autoavaliação do estado de saúde "ruim" em revisão sistemática da literatura, Brasil, 1999 a $2011^{a}$

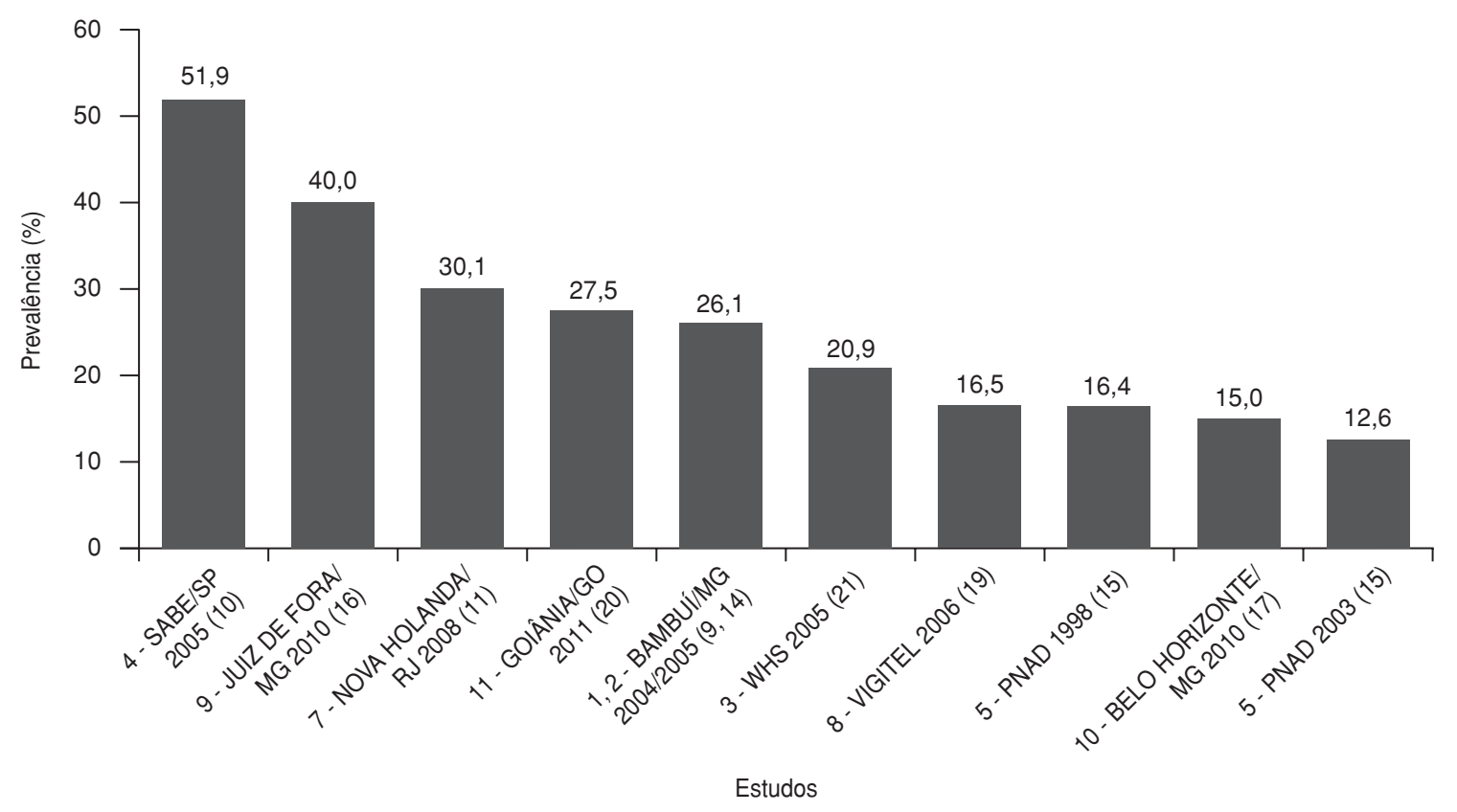

a Exceto Souza et al. (18), que não apresentaram um valor de prevalência geral.

brasileiras e o Distrito Federal, mostraram maior prevalência de autoavaliação ruim da saúde em pessoas com mais de 60 anos em comparação a faixas etárias jovens, nas mulheres das regiões Norte e Nordeste em comparação aos homens e entre os homens na Região Sul em comparação às mulheres. Essas diferenças de prevalência entre regiões podem estar relacionadas a aspectos socioeconômicos e demográficos. No Nordeste, a esperança de vida ao nascer em 2009 era de 70,5 anos, contra 74,7 no Sudeste. O produto interno bruto (PIB) per capita também apresenta variações significativas: 8 167,74 reais no Sudeste contra 22 147,13 reais no Sudeste (22). Esses indicadores evidenciam que as diferenças regionais refletem as desigualdades existentes no país em termos sociais e de saúde.

De forma geral, a prevalência de saúde autoavaliada como ruim foi elevada nos estudos, superior a 25\%. Quando se compara a prevalência de autoavaliação negativa de saúde no estudo de Souza et al. (18) em relação aos demais, observa-se que o valor médio da prevalência é de $25,5 \%$, o que é inferior ao Estudo SABE $(51,9 \%)(10)$ e superior à PNAD $(12,6 \%)$ (15). Na Região Centro-Oeste (20), cuja população foi de idosos usuários do SUS, a prevalência também foi elevada.
Não há na literatura valores de referência definindo o que seria aceitável em termos de saúde autoavaliada em idosos; assim, resta comparar os resultados de diferentes artigos e analisar os fatores associados similares e divergentes.

Quanto às características da pergunta e às opções de resposta, não há padronização. No estudo da autoavaliação negativa, as categorias de resposta "muito ruim" e "ruim" foram agrupadas. Contudo, utilizaram-se diversas técnicas de avaliação e arranjos entre as subcategorias, o que dificulta a comparação dos resultados. Nos estudos nos quais a prevalência de autoavaliação negativa foi maior do que 30\%, as categorias de resposta "regular" ou "razoável" podem ter contribuído para superestimar essa prevalência, pois foram as opções mais frequentes entre os idosos $(10,11,15,17$, 19). A superestimativa pode ainda estar relacionada à obtenção da informação diretamente da pessoa ou de terceiros, às diferenças no posicionamento da questão no questionário, bem como a limitações no delineamento dos estudos e nos ajustes na análise $(3,6,15,19)$.

Em relação à análise dos fatores socioeconômicos associados à autoavaliação ruim da saúde, destacam-se a baixa renda domiciliar mensal e a baixa classe econômica, encontradas em 54,5\% dos estudos. Essas variáveis têm importância marcante nos estudos, pois refletem o cenário de desigualdades sociais no Brasil. A baixa renda dos idosos atua negativamente no comportamento saudável, no ambiente domiciliar e no acesso aos serviços de saúde, mesmo quando disponibilizados adequadamente (23). Os idosos mais pobres procuram menos os serviços de saúde, possuem baixa adesão aos tratamentos e têm pouco acesso aos medicamentos, o que tem impacto direto nas condições de saúde do indivíduo (24). A autoavaliação do estado de saúde melhora conforme o aumento das condições socioeconômicas e o nível de informação das pessoas. A escolaridade, entretanto, foi associada à autoavaliação de saúde ruim em apenas um estudo, sugerindo a inserção desta variável em futuras investigações.

As variáveis referentes às condições de saúde foram predominantemente associadas à autoavaliação negativa de saúde dos idosos, destacando-se principalmente a polifarmácia e a presença de doenças. Embora o uso de múltiplos fármacos seja necessário para o controle das doenças, essa prática pode contribuir para alterações clínicas (25) que resultam em uma pior condição e 
percepção da saúde. Já a presença de doenças é uma variável cuja associação com autoavaliação de saúde negativa é esperada, podendo ser resultado do referencial de saúde dos idosos como ausência de doença. Sabe-se que as comorbidades podem trazer limitações e dificuldades de autocuidado (15), o que pode influenciar a percepção de saúde. Embora essa associação seja frequente, no Estudo SABE (10) os idosos que apresentaram de duas a três doenças crônicas tiveram menor probabilidade de avaliar sua saúde como ruim. Uma possível explicação para isso seria o fato de essas doenças estarem controladas (10).

Apesar da contribuição das comorbidades para uma percepção ruim, segundo Paskulin e Vianna (26), a percepção de ser doente está mais relacionada às incapacidades do que a ser portador de doenças crônicas. Ou seja, a saúde no idoso é determinada de forma mais adequada pela capacidade que ele possui para executar atividades cotidianas, ou seja, à sua capacidade funcional (27). Embora seja considerada paradigma para a saúde do idoso (27), a capacidade funcional foi uma variável de exposição pouco estudada. No Estudo SABE (10), quanto maior a incapacidade, maior a chance do idoso de autoperceber sua saúde como ruim, constituindo-se o principal determinante da autoavaliação negativa de saúde. Já no estudo Bambuí (9), a capacidade funcional, como variável de exposição, não foi associada.

Variáveis relativas ao acesso ao serviço de saúde, como hospitalizações e número de consultas médicas, apresentaram associação com autopercepção ruim de saúde (9, 14-16). Esses fatores podem contribuir para uma visão negativa, uma vez que a utilização do serviço não é garantia de resolutividade das demandas. No que se refere às hospitalizações, a restrição no ambiente hospitalar provoca impacto funcional no idoso, comprometendo o desempenho de suas atividades cotidianas e resultando numa auto-percepção ruim do estado de saúde (28).

Os achados deste estudo indicam elevada prevalência de autoavaliação negativa do estado de saúde em idosos brasileiros, variando de 12,6 a 51,9\%. Além disso, constatamos que as opções de resposta à pergunta sobre a autoavaliação do estado de saúde não são homogêneas, o que dificulta a comparação de resultados. Quanto aos fato- res associados, observa-se que há uma heterogeneidade entre os estudos, em função das variáveis investigadas, o que demonstra que a autoavaliação de saúde é influenciada não apenas pela presença da doença, suas causas e consequências, mas também pelo bem-estar, nível de satisfação com a vida, capacidade funcional e qualidade de vida, configurando-se como um importante indicador para ser monitorado nos serviços de saúde. A heterogeneidade de fatores associados indica que a saúde dos idosos é definida por determinantes que se aproximam do conceito ampliado de saúde.

Diante dos achados desta revisão sistemática, recomenda-se, na perspectiva da América Latina, o desenvolvimento de estudos sobre esta temática com idosos em diferentes cenários; agrupamento das categorias "ruim" e "muito ruim" para indicar uma autoavaliação negativa; análise separada da categoria "regular"; agrupamento das categorias "boa" e "muito" boa para análise da dimensão positiva; investigação de variáveis ainda pouco exploradas, como capacidade funcional, acesso aos serviços, variáveis contextuais e de suporte social. Tendo em vista a ausência de valores de referência

TABELA 3. Fatores associados à auto-avaliação do estado de saúde ruim nos estudos desenvolvidos com idosos brasileiros, 1999 a $2011^{a}$

\begin{tabular}{|c|c|c|c|}
\hline $\begin{array}{c}\text { Código } \\
\text { (referência) }\end{array}$ & Variáveis de exposição & Variáveis associadas ao desfecho & $\begin{array}{l}\text { Tipo de } \\
\text { análise }\end{array}$ \\
\hline $1(9)$ & $\begin{array}{l}\text { - Demográficas (faixa etária, sexo, escolaridade, renda domiciliar mensal, } \\
\text { estado conjugal, no. de gerações morando no mesmo domicílio) } \\
\text { - Suporte social (frequência a clubes, associações, cultos religiosos, } \\
\text { satisfação com relacionamentos pessoais) } \\
\text { - Estilo de vida (fumante atual, consumo de álcool nos últimos } 12 \text { meses, } \\
\text { exercícios físicos no período de lazer, consumo diário de frutas e vegetais } \\
\text { frescos) } \\
\text { - Condições de saúde (incapacidade de realizar atividades de vida diária, } \\
\text { sintomas depressivos e ansiosos, insônia, síndrome metabólica, presença } \\
\text { de anticorpos Trypanossoma cruzi, número de medicamentos prescritos e } \\
\text { não prescritos usados nos últimos } 90 \text { dias) }\end{array}$ & $\begin{array}{l}\text { - Baixa renda domiciliar mensal } \\
\text { - Insatisfação/ muita insatisfação com os } \\
\text { relacionamentos pessoais } \\
\text { - Sintomas depressivos e ansiosos nas últimas } \\
2 \text { semanas } \\
\text { - Queixa de insônia nos últimos } 30 \text { dias } \\
\text { - Número de medicamentos prescritos usados nos } \\
\text { últimos } 30 \text { dias ( } 1-2,3-4 \text { e } \geq 5) \\
\text { - Queixa acerca de cuidados médicos (preço da } \\
\text { consulta, filas, outros) } \\
\text { - Número de consultas médicas nos últimos } \\
12 \text { meses }(2-3 \text { e } \geq 4) \\
\text { - Número de internações hospitalares nos últimos } \\
12 \text { meses }(\geq 2)\end{array}$ & $\begin{array}{l}\text { Regressão } \\
\text { logística } \\
\text { múltipla }\end{array}$ \\
\hline $2(14)$ & $\begin{array}{l}\text { - Sociodemográficas (idade, sexo, escolaridade, estado civil, no. de } \\
\text { gerações morando no mesmo domicílio, renda familiar mensal) } \\
\text { - Rede social (participação em igreja, clubes ou associações e satisfação } \\
\text { com a rede social) } \\
\text { - Estilo de vida (tabagismo, ingestão de álcool, lazer, atividade física e } \\
\text { consumo de frutas e legumes) } \\
\text { - Capacidade funcional (incapacidade para realizar pelo menos uma das } \\
\text { seguintes atividades da vida diária: tomar banho, vestir-se, passar da } \\
\text { cama para a cadeira, ir ao banheiro) } \\
\text { - Outros indicadores de saúde (sintomas depressivos, insônia, uso de } \\
\text { medicamentos) } \\
\text { - Acesso e utilização dos serviços de saúde (queixas quanto aos cuidados } \\
\text { médicos, número de visitas ao médico, número de internações) }\end{array}$ & $\begin{array}{l}\text { - Satisfação com a rede social (insatisfatório/ } \\
\text { insatisfatório) } \\
\text { - Sintomas depressivos } \\
\text { - No. de consultas médicas ( } 1,2-3 \text { e } \geq 4 \text { nos últimos } \\
12 \text { meses) } \\
\text { - No. de internações ( } \geq 2 \text { nos } 12 \text { meses precedentes) } \\
\text { - Insônia } \\
\text { - Soropositividade para T. cruzi, } \\
\text { - Número de medicamentos prescritos (1-2, 3-4 e } \geq 5 \\
\text { nos últimos } 90 \text { dias) } \\
\text { - Problemas quanto à assistência médica }\end{array}$ & $\begin{array}{l}\text { Regressão } \\
\text { logística } \\
\text { multivariada }\end{array}$ \\
\hline
\end{tabular}


TABELA 3. Continuação

\begin{tabular}{|c|c|c|c|}
\hline $\begin{array}{c}\text { Código } \\
\text { (referência) }\end{array}$ & Variáveis de exposição & Variáveis associadas ao desfecho & $\begin{array}{l}\text { Tipo de } \\
\text { análise }\end{array}$ \\
\hline $4(10)$ & $\begin{array}{l}\text { - Demográficas (idade, sexo, estado conjugal, arranjo familiar) } \\
\text { - Socioeconômicas (educação, renda) } \\
\text { - No. de doenças crônicas (hipertensão, artrite/reumatismo, doença } \\
\text { cardiovascular, diabetes, asma/bronquite/enfisema, embolia/AVC, câncer) } \\
\text { - Capacidade funcional (atravessar um quarto caminhando, alimentar-se, } \\
\text { deitar e levantar da cama, usar o vaso sanitário, vestir e despir, tomar } \\
\text { banho, preparar uma refeição quente, fazer tarefas domésticas leves, } \\
\text { fazer tarefas domésticas mais pesadas) }\end{array}$ & $\begin{array}{l}\text { - Sexo feminino } \\
\text { - Baixa renda } \\
\text { - Informante secundário } \\
\text { - Presença de doenças } \\
\text { - Capacidade funcional }\end{array}$ & $\begin{array}{l}\text { Regressão } \\
\text { logística binária } \\
\text { múltipla }\end{array}$ \\
\hline $5(15)$ & $\begin{array}{l}\text { - Social (escolaridade) } \\
\text { - Condições de saúde (no. doenças crônicas) } \\
\text { - Uso de serviços de saúde (no. consultas médicas realizadas nos últimos } \\
12 \text { meses e no. hospitalizações nos últimos } 12 \text { meses }\end{array}$ & $\begin{array}{l}\text { Idoso: } \\
\text { - } \text { escolaridade } \geq 8 \text { anos de estudos (fator de } \\
\text { proteção) } \\
\text { - } \geq 3 \text { doenças crônicas } \\
\text { - } \geq 1 \text { consultas médicas no último ano } \\
\text { - } \geq 2 \text { hospitalizações no último ano } \\
\text { Informante: } \\
\text { - } \geq 3 \text { doenças crônicas } \\
\text { - } \geq 1 \text { hospitalizações no último ano }\end{array}$ & $\begin{array}{l}\text { Regressão } \\
\text { logística } \\
\text { multivariada }\end{array}$ \\
\hline $7(11)$ & $\begin{array}{l}\text { - Informações sociodemográficas (idade, situação conjugal, escolaridade, } \\
\text { renda, tamanho da família) } \\
\text { - Morbidade referida }\end{array}$ & $\begin{array}{l}\text { - Faixa etária > } 80 \text { anos } \\
\text { - Renda familiar } \\
\text { - Trabalho } \\
\text { - Ter problemas de saúde } \\
\text { - Insatisfação com a vida }\end{array}$ & Qui-quadrado \\
\hline $9(16)$ & $\begin{array}{l}\text { - Demográficas (idade, escolaridade, fonte de renda, trabalho atual) } \\
\text { - Estilo de vida (tabagismo atual, prática de atividade física) } \\
\text { - Condições de saúde e uso do serviço (disponibilidade de serviço de } \\
\text { saúde de referência, uso regular de medicação, internação nos últimos } \\
12 \text { meses) } \\
\text { - Condições crônicas auto referidas (hipertensão arterial, colesterol e/ } \\
\text { ou triglicérides elevados, diabetes, doenças cardiovasculares, doença } \\
\text { respiratória crônica, doença renal crônica e depressão) }\end{array}$ & $\begin{array}{l}\text { - Condições crônicas de saúde, } \\
\text { - Uso de medicação regular } \\
\text { - Relato de internação recente } \\
\text { - Não trabalhar na ocasião da entrevista } \\
\text { - Escolaridade menor que o fundamental } \\
\text { - Usuário do serviço público de saúde }\end{array}$ & $\begin{array}{l}\text { Multivariada } \\
\text { por regressão } \\
\text { de Poisson }\end{array}$ \\
\hline $10(17)$ & $\begin{array}{l}\text { - Sociodemográficas (sexo, faixa etária, estado civil, cor, renda familiar } \\
\text { mensal, escolaridade, trabalho na semana anterior) } \\
\text { - Estilo de vida (fumante atual, consumo de álcool nos últimos } 30 \text { dias, } \\
\text { prática de atividade física no lazer, consumo de frutas e verduras frescas } \\
\text { nos últimos } 7 \text { dias) } \\
\text { - Condições de saúde (no, doenças crônicas, dificuldade ou incapacidade } \\
\text { nas atividades de vida diária) } \\
\text { - Uso dos serviços de saúde (procurar o mesmo lugar quando precisa } \\
\text { de cuidados médicos, consulta com médico nos últimos } 12 \text { meses, } \\
\text { hospitalização nos últimos } 12 \text { meses, cobertura por plano de saúde) }\end{array}$ & $\begin{array}{l}\text { Idoso: } \\
\text { - Dificuldade/incapacidade para realizar atividades } \\
\text { de vida diária } \\
\text { - Faixa etária } 65 \text { a } 69 \text { anos } \\
\text { Informante: } \\
\text { - Presença de doenças crônicas } \\
\text { - Dificuldade/incapacidade para realizar atividades } \\
\text { de vida diária }\end{array}$ & $\begin{array}{l}\text { Análise } \\
\text { multivariada }\end{array}$ \\
\hline $11(20)$ & $\begin{array}{l}\text { - Demográficas e socioeconômicas (sexo, idade, número de residentes no } \\
\text { domicílio, estado civil, cor da pele, escolaridade, classe econômica) } \\
\text { - Estilo de vida (tabagismo, consumo de bebidas alcoólicas, prática de } \\
\text { atividade física, consumo diário de frutas e vegetais) } \\
\text { - Condições de saúde (morbidades referidas, número de morbidades, perda } \\
\text { de peso recente, índice de massa corporal, número de medicamentos em } \\
\text { uso, internação no último ano) }\end{array}$ & $\begin{array}{l}\text { - Escolaridade }<1 \text { ano de estudo } \\
\text { - Não praticar atividade física } \\
\text { - Uso de cinco ou mais medicamentos } \\
\text { - Perda de peso recente }\end{array}$ & $\begin{array}{l}\text { Análise } \\
\text { multivariada } \\
\text { por } \\
\text { regressão } \\
\text { de Poisson } \\
\text { hierarquizada }\end{array}$ \\
\hline
\end{tabular}

\footnotetext{
a Três artigos $(21,18,19)$ não aparecem na tabela porque apresentaram os fatores associados à autoavaliação de saúde ruim apenas para a população geral, sem estratificação por faixa etária.
}

considerados aceitáveis, recomenda-se, ainda, o valor máximo de $25 \%$ para a prevalência de autoavaliação de saúde ruim entre idosos. Prevalências de autoavaliação negativa acima desse valor devem suscitar uma avaliação específica de determinantes por profissionais de saúde para identificar medidas de reversão ou minimização, envolvendo políticas públicas de saúde, educação permanente de profissionais e políticas sociais.

Em função do crescimento da população idosa e da aplicabilidade da autoavaliação do estado de saúde como indicador das condições gerais de saúde da população, pesquisas sobre esta te- mática são bem-vindas e possibilitarão conhecer, acompanhar e comparar resultados para orientar a tomada de decisão no tocante à formulação de políticas de saúde para o Brasil e a América Latina.

Conflitos de interesse. Nada declarado pelos autores. 


\section{REFERÊNCIAS}

1. Sadana R, Mathers CD, Lopez AD, Murray $\mathrm{CJL}$, Iburg KM. Comparative analyses of more than 50 household surveys on health status. Em: Murray CJL, Salomon JA, Mathers CD, Lopez AD. Summary measures of population health: concepts, ethics, measurement and applications. Genebra: WHO; 2002. Pp. 369-86.

2. Barros MB. Auto-avaliação de saúde. Em: Cesar CLG, Carandina L, Alves MCGP, Barros MB, Goldbaum M. Saúde e condição de vida em São Paulo - Inquérito multicêntrico de saúde em São Paulo - ISA/SP. São Paulo: FSP/USP; 2005. Pp. 153-62.

3. Damian J, Ruigomez A, Pastor V, MartinMoreno JM. Determinants of self assessed health among Spanish older people living at home. J Epidemiol Community Health. 1999;53(7):412-6.

4. Phillips LJ, Hammock RL, Blanton JM. Predictors of self-rated health status among Texas residents. Prev Chronic Dis. 2005;2(4):1-10.

5. Ramkumar A, Quah JL, Wong T, Yeo LS, Nieh CC, Shankar A, et al. Self-rated health, associated factors and diseases: community-based cross-sectional study of Singaporean adults aged 40 years and above. Ann Acad Med Singapore. 2009;38(7):606-7.

6. Dachs JNW. Determinantes das desigualdades na auto-avaliação do estado de saúde no Brasil: análise dos dados da PNAD/1998. Cienc Saude Coletiva. 2002;7(4):641-57.

7. Kikuchi EL. Auto-avaliação da Saúde. Em: Jacob Filho W. Avaliação global do idoso. Manual da Liga do GAMIA. São Paulo: Editora Atheneu; 2005. Pp. 45-9.

8. Closs EV, Schwanke CHA. A evolução do índice de envelhecimento no Brasil, nas suas regiões e unidades federativas no período de 1970 a 2010. Rev Bras Geriatr Gerontol. 2012;15(3):443-458.

9. Lima-Costa MF, Firmo JOA, Uchôa EA. A estrutura da auto-avaliação da saúde entre idosos: projeto Bambuí. Rev Saude Publica. 2004;38(6):827-34

10. Alves LC, Rodrigues RN. Determinantes da autopercepção de saúde entre idosos do $\mathrm{Mu}$ nicípio de São Paulo, Brasil. Rev Panam Salud Publica. 2005;17(5/6):333-41.
11. Sousa AI, Silver LD. Perfil sociodemográfico e estado de saúde auto-referido entre idosas de uma localidade de baixa renda. Esc Anna Nery Rev Enferm. 2008;12(4):706-16.

12. Idler EL, Benyamini Y. Self-rated health and mortality: a review of twenty-seven community studies. J Health Soc Beh. 1997;38(1): 21-37.

13. Moher D, Liberati A, Tetzlaff J, Altman DG, PRISMA Group. Preferred reporting items for systematic reviews and meta-analyses: the PRISMA statement. Ann Intern Med. 2009; 151(4):264-9.

14. Lima-Costa MF, Firmo JOA, Uchôa E. Differences in self-rated health among older adults according to socioeconomic circumstances: the Bambuí Health and Aging Study. Cad Saude Publica. 2005;21(3):830-9.

15. Lima-Costa MF, Peixoto SV, Matos DL, Firmo JOA, Uchôa E. A influência de respondente substituto na percepção de saúde de idosos: um estudo baseado na Pesquisa Nacional por Amostra de Domicílios $(1998,2003)$ e na coorte de Bambuí, Minas Gerais, Brasil. Cad Saude Publica. 2007a;23(8):1893-902.

16. Santiago LM, Novaes CO, Mattos IE. Factors associated with self-rated health among older men in a medium-sized city in Brazil. J Men's Health. 2010;7(1):55-63.

17. Jardim R, Barreto SM, Giatti L. Self-reporting and secondary informant reporting in health assessments among elderly people. Rev Saude Publica 2010;44(6):1120-9.

18. Souza MC, Otero UB, Almeida LM, Turci SRB, Figueiredo, VC, Lozana JA. Auto-avaliação de saúde e limitações físicas decorrentes de problemas de saúde. Rev Saude Publica. 2008; 42(4):741-9.

19. Barros MBA, Zanchetta LM, Moura EC, Malta DC. Auto-avaliação da saúde e fatores associados, Brasil, 2006. Rev Saude Publica. 2009; 43 Suppl 2:S27-37.

20. Pagotto V, Nakatani AYK, Silveira EA. Fatores associados à autoavaliação de saúde ruim em idosos usuários do Sistema Único de Saúde. Cad Saude Publica. 2011; 27(8):1593-1602.

21. Szwarcwald CL, Souza-Júnior PRB, Esteves MAP, Damacena GN, Viacava F. Socio- demographic determinants of self-rated health in Brazil. Cad Saude Publica. 2005;21 suppl: S54-64.

22. Instituto Brasileiro de Geografia e Estatística (IBGE). Indicadores sociodemográficos e de saúde no Brasil. Rio de Janeiro: IBGE; 2009. (Estudos e Pesquisas - Informação Demográfica e Socioeconômica número 25.) Disponível em: http://www.ibge.gov.br/ home/estatistica/populacao/indic socio saude/2009/ Acessado em abril de 2013.

23. Travassos C, Viacava F, Laguardia J. Os Suplementos Saúde na Pesquisa Nacional por Amostra de Domicílios (PNAD) no Brasil. Rev Bras Epidemiol. 2008;11(suppl 1):98-112.

24. Lima-Costa MF, Barreto SM, Firmo JOA, Uchôa E. Socioeconomic position and health in a population of Brazilian elderly: the Bambuí Health and Aging Study (BHAS). Rev Panam Salud Publica. 2003; 13(6):387-94

25. Medeiros-Souza P, Santos-Neto LL, Kusano LTE, Pereira MG. Diagnosis and control of polypharmacy in the elderly. Rev Saude Publica. 2007;41(6):1049-53.

26. Paskulin LMG, Vianna LAC. Perfil sociodemográfico e condições de saúde auto-referidas de idosos de Porto Alegre. Rev Saude Publica. 2007;41(5):757-68.

27. Ramos LR. Fatores determinantes do envelhecimento saudável em idosos residentes em centro urbano: Projeto Epidoso, São Paulo. Cad Saude Publica. 2003;19(3):793-7.

28. Siqueira $A B$, Cordeiro $R C$, Perracini $M R$, Ramos LR. Impacto funcional da internação hospitalar de pacientes idosos. Rev Saude Publica. 2004;38(5):687-94.

Manuscrito recebido em 27 de março de 2012. Aceito em versão revisada em 29 de novembro de 2012. 
ABSTRACT Objective. To conduct a systematic review of the literature on self-assessment of health status in the elderly population.

Self-assessment of health by older Brazilians: systematic review of the literature

Key words
Methods. Medline and LILACS were searched following the Reporting Items for Systematic Reviews and Meta-Analyses (PRISMA) methodology. Original articles on self-assessment of health status conducted with older Brazilians were selected. The following were examined: general characteristics of the studies, the prevalence of negative self-assessment of health, the factors associated with this negative selfassessment, the question used to inquire about health status and response categories. Results. Of 97 studies identified, 11 met the inclusion criteria. Variations between the studies regarding the formulation of the question and answer choices were identified. The prevalence of negative health self-assessment ranged from 12.6 to $51.9 \%$ between studies. The most usual dependent variables associated with negative selfassessment of health were the presence of disease, number of medications, monthly family/household income, hospitalizations, medical office visits, difficulty/inability to perform activities of daily living, presence of depressive and anxiety symptoms, and complaints of insomnia.

Conclusions. The heterogeneity of factors associated with a negative self-evaluation indicates that the health of older individuals is defined by determinants that fall within a broad view of health. The standardization of questions and answers for research on self-assessment of health in older people is recommended, since this information will produce knowledge and allow monitoring and comparison of results, and will therefore be useful in guiding decision-making regarding the formulation of health policies for Brazil and Latin America.

Aged; self-assessment; health status; review; Brazil. 